\title{
Response to Comment on: "Growth, Optical, Thermal, Mechanical, Laser Damage Threshold and Electrical Polarizability of Cadmium Chloride Doped L-Alanine (LACC) Single Crystal for Optoelectronic Applications" [J. Electron. Mater., 48, 7915-22 (2019)]
}

\author{
C. Karnan ${ }^{1}$
}

Received: 15 August 2020 / Accepted: 17 March 2021 / Published online: 15 April 2021

(c) The Minerals, Metals \& Materials Society 2021

Concerning the above, I would like to submit my response. The author stated that the absorption coefficient, reflectance and refractive index were incorrect. We made a typographical error in the absorption coefficient formula, and the correct formula can be verified from the reference cited in our work. There was a mistake in the formula write-up in our manuscript because the reference paper formula was wrong, and the same formula was repeated in my paper. I accept the error in the write-up, but for the calculation, we used the correct formula. The formula which is used to calculate reflectance $(R)$ and refractive index $(n)$ is

$R=\frac{\exp (-\alpha t)-\sqrt{\exp (-\alpha t) T-\exp (-3 \alpha t) T+\exp (-2 \alpha t) T^{2}}}{\exp (-\alpha t)+\exp (-2 \alpha t) T}$
$n=\frac{-(R+1+2 \sqrt{R)}}{(R-1)}$

So the formula which we used to calculate is similar to the author comment (supplementary file). Here there is no mistake in calculated values, only mistakes in the formula write-up.

Supplementary Information The online version contains supplementary material available at https://doi.org/10.1007/s11664-021-08891-y.

Publisher's Note Springer Nature remains neutral with regard to jurisdictional claims in published maps and institutional affiliations.

C. Karnan

c.karnan@yahoo.com; karnan.phy@drmgrdu.ac.in

1 Department of Physics, Dr. M.G.R. Educational and Research Institute, Chennai 600 095, India 